\title{
STUDY ON CONTACT BETWEEN DOUBLE METAL COMPOSITE AND PIPE POWER SLIP FOR OFFSHORE WELL
}

\author{
Wang Yu, Dr., \\ Ai Zhijiu, Prof., \\ School of Mechatronic Engineering, Southwest Petroleum University, Chengdu, Sichuan, China \\ Shi Changshuai, Dr. \\ Key Laboratory of Fluid and Power Machinery, Ministry of Education (XIHUA University) Chengdu, Sichuan, China \\ Ai Yu, Engineer., \\ CAAC, Southwest Air Traffic Management Bur, Chengdu, Sichuan, China
}

\begin{abstract}
The whole process of clamping offshore well double metal composite pipe was established, Reviewing the problems that in the oil and gas wells the double metal composite pipes frequently get plastic damage or even fracture failure when the power slips hold fast to the double metal composite pipe, the effect Eq. of contact area to tooth plate and double metal composite pipe was deduced, and based on the elastoplastic contact analysis theory, the theoretical Eq. was verified. For the double metal composite pipe stress, displacement and stress of drill tooth plate, influence of slip teeth plate on the double metal composite pipe damage was analyzed, The plastic damage law of the double metal composite pipe was obtained with the influence factor of the contact surface and the influence factor of the tooth crown width. the analysis showed that, The double metal composite pipe can be clamped and the contact area value of the tooth plate with the minimum damage are fixed. Several sets of teeth were produced, and then used to carry out experiments, indoor and field experiments have fully proved the accuracy of the result, The research work provides a better theoretical basis for optimal design of slip tooth plate.
\end{abstract}

Keywords: Power slip; Double metal composite pipe; Contact area; Slip teeth; Plastic damage

\section{INTRODUCTION}

Due to the complex situation of the underground offshore well, in order to prevent corrosion and meet the strength requirements, double metal composite pipe was widely used in offshore wells. The compressive strength of double metal composite pipe lining was very low, therefore, when the pipe was clamped, the stress of the pipe and the clamping tool should be focused, the slip was used to clamp the offshore well pipe as a kind of commonly used and important tool, which is used very high frequency. In the use of the slip, slip is mosaic in the middle of the rotary disc, clinging to the outer surface of the double metal composite pipe, under the action of gravity, double metal composite pipe will slide down, due to the effect of friction and pressure, slip will slide down with the double metal composite pipe sliding, in this process, constrained by the inner diameter of the turntable, slip is gradually radial shrinkage and card tight double metal composite pipe. Field investigation found that the drilling safety and cost will be generated by a greater impact because of the slip teeth plate, at present, in the process of clamping double metal composite pipe, the damage of double metal composite pipe surface will be generated, which reduces the drilling capacity, and it is one of the causes of pipe failure in the drilling process. Finally, because of the delay of drilling and drilling tool failure, huge economic losses will be produced $[1,2,3]$.

The tooth is a key part of slip, which contacts directly with the double metal composite pipe, The extent of double 
metal composite pipe damage and whether the double metal composite pipe is clamped is determined by tooth shape parameter of tooth plate[4]. In the work, the clamping force is determined by the slip teeth in the tooth profile parameters, force is too small, double metal composite pipe will decline, the force is too large, the double metal composite pipe and the tooth plate will occur larger plastic deformation, resulting in failure[5]. Slip technologically advanced is United States of DEN-CON company and VARCO-BJ company, which ppipeuced a slip of the double metal composite pipe small damage, the other slips for drill damage was relatively large, as shown in Figure 1. Thus, reducing the slips for double metal composite pipe plastic damage is an issue on oil and gas fields need to be resolved, and dental plate contact area with the double metal composite pipe size is one of the key factors affecting the double metal composite pipe slips injury.

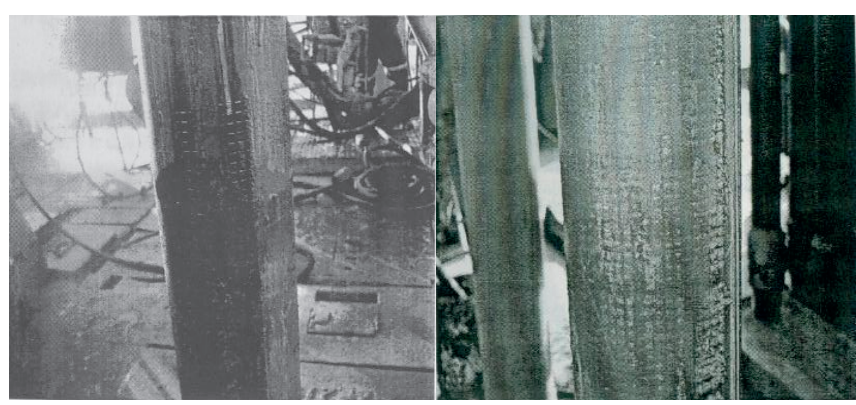

Fig. 1. Injury for offshore well pipe

Currently the paper of researching slips dental plate is relatively small, which mainly concentrated in the mechanical analysis of the entire system, the double metal composite pipe and slip damage mechanism on the double metal composite pipe. Hongliang Tian carried out experimental study for the coefficient of friction between the slips and the drill, who obtained changing scope and value of grooved slip friction coefficient, and clearly put forward the axial grooves, lateral grooves and slip teeth Board tooth surface will affect the coefficient of friction[6]; Weiliang Liu studied the affection of different tooth surface for the impact force, proposed reducing clamping system preload by changing the structure and shape of the tooth surface slip for increasing the friction coefficient method, in order to stabilize the double metal composite pipe clamping system[7]; Wang Yong, who analyzed the anti-failure mechanism of dynamic slip gripping zone, and further proposed measures to prevent drilling failure in the slip zone[8]; WHSPiri and WBReinhold studied the mechanism of damaging drilling by slip, and analyzed the double metal composite pipe bite injuries on several factors, such as slip tooth dental plate, pipe load[9,10]. But the work of the specific studying a tooth dental plate slips parameters affect for the drill has not yet commenced, and the affection of changing each slip tooth plate parameters on drill plastic damage is particularly large, in this paper, the double metal composite pipe damage for the contact area was systematically studied, since the contact area slips dental plate and double metal composite pipe slips directly determines the size of the hold force and friction, the work is essential to the use of slip, which is one of the key challenges of the double metal composite pipe slips injury[11,12].

\section{CONTACT SIMULATION MODEL}

\section{SLIP DENTAL PLATE STRESS ANALYSIS}

When offshore well slip was working, the double metal composite pipe was clamped by slip, as shown in Fig. 2. According to most scholars of research, we also assumes a uniform distribution of radial pressure, the coefficient of friction between the slips and drill the same everywhere[13].

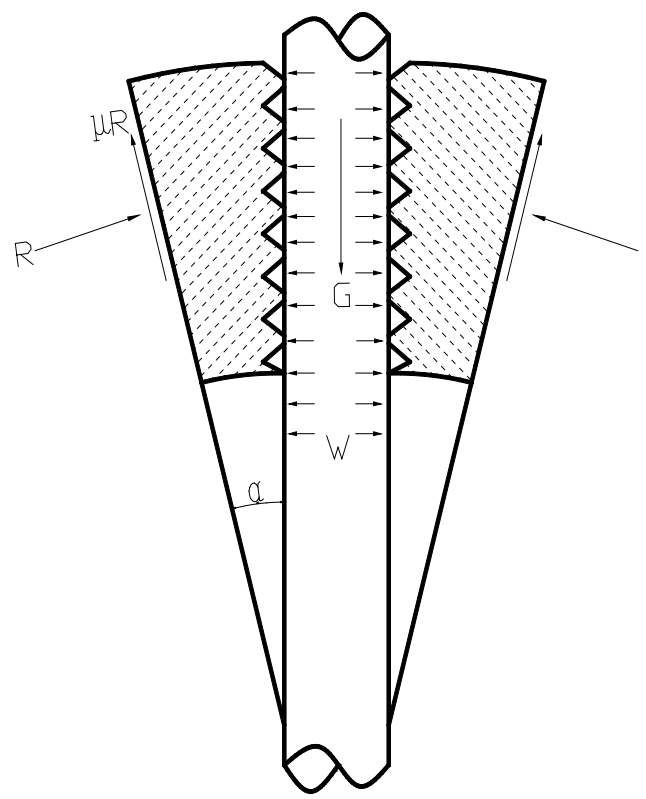

Fig. 2. Contact model between double metal composite pipe and slip

In Fig. 2, $\mathrm{R}$ is extrusion pressure, $\mu \mathrm{R}$ is friction; $\mathrm{G}$ is the gravity of double metal composite pipe, $\mathrm{W}$ is reaction force of double metal composite pipe; $\alpha$ is the angle between the slip outer surface and double metal composite pipe outer surface. These parameters were meet the following Eq.[9].

$$
\left\{\begin{array}{l}
W=R(\cos \alpha-\mu \sin \alpha) \\
G=R(\sin \alpha+\mu \cos \alpha)
\end{array}\right.
$$

Futher:

$$
W=G(1-\mu \tan \alpha) /(\mu+\tan \alpha)
$$

As shown in Fig. 3, the overall tooth plate is on the left, the right is an enlarged single tooth, $L$ represents the total length of the dental lamina, $l$ represents the tooth top width, $h$ represents the tooth height, $\beta$ represents tooth rake angle, $\gamma$ represents tooth posterior horn. 


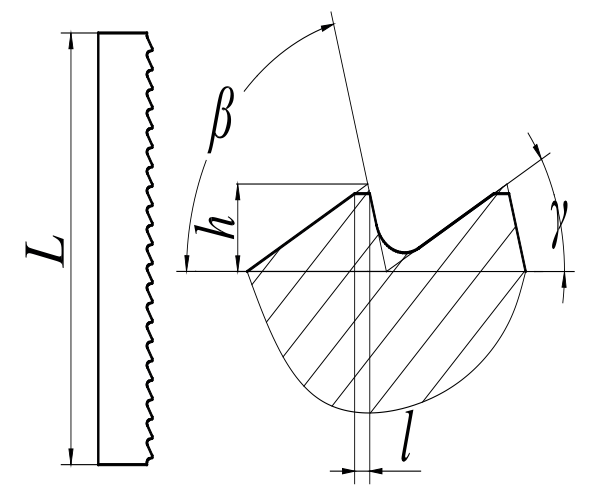

Fig. 3. Slip teeth schematic

The number of teeth can be calculated from Fig. 3:

$$
n=\frac{L}{h(1 / \tan \beta+1 / \tan \gamma)}
$$

The follow is surface area of each tooth addendum:

$$
s=\frac{l \pi r \phi}{180}
$$

Thus, when slip was clamping double metal composite pipe, slip tooth stress was generated as follows:

$\sigma=\frac{W}{S}=\frac{W}{m n s}=\frac{180 G h(\tan \beta+\tan \gamma)(1-\mu \tan \alpha)}{m \pi r l \phi L(\mu+\tan \alpha) \tan \beta \tan \gamma}$

In Eq. (5):

$S$ - the total area of slip teeth plate in contact with the double metal composite pipe;

$\Phi \quad$ - the angle of tooth plate rotation;

$m$ - the number of teeth.

Assumed:

$$
\begin{gathered}
A=\frac{180 h(\tan \beta+\tan \gamma)}{\phi L \tan \beta \tan \gamma} \\
B=\frac{1-\mu \tan \alpha}{\mu+\tan \alpha}
\end{gathered}
$$

Therefore, we also obtained:

$$
\sigma=A B \frac{G}{m \pi r l}
$$

In Eq. (8):

$A$ - the contact surface factor;

$B-$ a radial load factor.

In the design of slip tooth plate, the stress was required that the smaller the better, from the Eq. (8), we can find that, in case of determined the tooth affecting factors of contact area, the larger for teeth tooth top width l, less for stress of tooth plate, the stress on the double metal composite pipe is also smaller. However, when the stress is too small, the friction between the tooth plate and drill is not sufficient to hold the hanging double metal composite pipe, double metal composite pipe slips tooth plate and critical contact friction is determined by Coulomb friction law[11]:

$$
\tau_{c r}=\mu_{1} p
$$

In Eq. (9):

$\mu_{1}$ - friction between slip tooth plate and double metal composite pipe;

$p$ - the normal contact stress.

Binding Eq. (5) and Eq. (9), the frictional resistance in clamping double metal composite pipe by slip can be generated:

$F_{f}=\mu_{1} \sigma=\mu_{1} \frac{W}{S}=\mu_{1} \frac{W}{m n s}=\frac{180 G h \mu_{1}(\tan \beta+\tan \gamma)(1-\mu \tan \alpha)}{m \pi r l \phi L(\mu+\tan \alpha) \tan \beta \tan \gamma}$

The greater the friction, the greater the clamping force, thus, by the above Eq., we can obtain that, in case of determined the tooth affecting factors of contact area, the larger for teeth tooth top width $l$, more conducive for slip working. So we saw that from Eq. (5) and Eq. (10), there will be an optimum tooth top width, it is not only reducing the stress when the slip working, but also to generating sufficient friction resistance force in order to clamp double metal composite pipe by slip.

\section{DOUBLE METAL COMPOSITE PIPE STRESS ANALYSIS}

In the process of holding the double metal composite pipe by slip, assuming no friction angle, the follow is the axial, radial, circumferential stress $\sigma_{x}, \sigma_{r}, \sigma_{\theta}$, respectively[10]

$$
\begin{gathered}
\sigma_{x}=G / S_{H} \\
\sigma_{r}=\frac{w r_{o}^{2}}{r_{o}^{2}-r_{i}^{2}}\left(1-\frac{r_{i}^{2}}{r^{2}}\right) \\
\sigma_{\theta}=\frac{w r_{o}^{2}}{r_{o}^{2}-r_{i}^{2}}\left(1+\frac{r_{o}^{2}}{r^{2}}\right)
\end{gathered}
$$

In these Eq.s:

$$
w=W / S_{L}
$$




$$
S_{H}=\pi\left(r_{o}^{2}-r_{i}^{2}\right)
$$

In Eq. (14) and Eq. (15):

$S_{H}$ - the pipe cross-sectional area;

$w$ - double metal composite pipe extrusion stress;

$r_{o}$ - the outer diameter of the double metal composite pipe;

$r_{i}$ - the inner diameter of the double metal composite pipe;

$S_{L}-$ the side slip area.

We can obtain that, when the double metal composite pipe was clamped, frictional resistance of the outer surface of the double metal composite pipe slips generated as follows:

$f_{F}=\int_{0}^{m} \int_{0}^{n} \int_{0}^{l} \mu_{1} \sigma_{r} d x d y d z=\int_{0}^{m} \int_{0}^{n} \int_{0}^{l} \mu_{1} \frac{w r_{o}^{2}}{r_{o}^{2}-r_{i}^{2}}\left(1-\frac{r_{i}^{2}}{r_{o}^{2}}\right) d x d y d z$

The double metal composite pipe stress is generated by gravity:

$$
f_{G}=\int_{0}^{a} \frac{G}{\pi\left(r_{o}^{2}-r_{i}^{2}\right)} d x
$$

In Eq. (17):

$a$ - the length of the double metal composite pipe.

In order to be able to clamp the drill, it must be $f_{F}=f_{G}$. United (16) (17):

$$
\pi m L l \mu_{1} w\left(r_{o}^{2}-r_{i}^{2}\right) \tan \beta \tan \gamma=G a h(\tan \beta+\tan \gamma)
$$

The Eq. can be written as:

$$
l=C \frac{G a}{\pi m \mu_{1} w\left(r_{o}^{2}-r_{i}^{2}\right)}
$$

In Eq. (19):

$C$ - the tooth top width factor.

$$
C=\frac{h(\tan \beta+\tan \gamma)}{L \tan \beta \tan \gamma}
$$

Therefore, for different tooth dental plate parameters, the optimal parameter with the tooth was calculated by the Eq. (19), for example, after the tooth rake angle, tooth posterior horn and tooth height were determined, we can calculate the optimal tooth top width, Then, Bring parameters into the Eq. (8), get the best of kava dental plate.

The following Eq. is the double metal composite pipe failure criteria, $\sigma_{s}$ is the double metal composite pipe yield strength.

$$
\left(\sigma_{x}-\sigma_{r}\right)^{2}+\left(\sigma_{x}-\sigma_{\theta}\right)^{2}+\left(\sigma_{r}-\sigma_{\theta}\right)^{2}=2 \sigma_{s}^{2}
$$

The drill lateral and medial can be calculated by the above Eq.:

$$
\sigma_{o .}=G S_{L} \sigma_{s} \sqrt{\frac{2}{\left(G S_{L}+W \pi r_{o}^{2}-W \pi r_{i}^{2}\right)^{2}+\left(G S_{L}+W \pi r_{o}^{2}+W \pi r_{i}^{2}\right)^{2}+4 W^{2} \pi^{2} r_{i}^{4}}}
$$

$$
\sigma_{i}=G S_{L} \sigma_{s} \sqrt{\frac{2}{\left(G S_{L}+W \pi r_{o}^{2}-W \pi r_{i}^{2}\right)^{2}+\left(G S_{L}+2 W \pi r_{o}^{2}\right)^{2}+4 W^{2} \pi^{2} r_{o}^{4}}}
$$

If the force is too small to hold fast, it will lead to drill decline. And the double metal composite pipe also occurs decline phenomenon, this displacement consists of two parts, one part is drill axial tension generated by the displacement $s_{1}$, the other part is the displacement of the double metal composite pipe to the pressure generated by the ring $s_{2}$. Specific expressions are as follows[12]:

$$
\begin{gathered}
s_{1}=-r \varepsilon \\
s_{2}=\frac{G}{16 \pi r \lambda^{4} D}\left[2-(\cos \lambda x) \mathrm{e}^{-\lambda x}-\mathrm{e}^{-\lambda(b-x)} \cos \lambda(b-x)\right]
\end{gathered}
$$

$$
\lambda=\frac{\sqrt[4]{3\left(1-\mu^{2}\right)}}{\sqrt{r c}}
$$

In Eqs. (24), (25), (26):

$\varepsilon$ - the strain drill;

$D$ - the flexural rigidity of the double metal composite pipe;

$x$ - the contact length between slip and double metal composite pipe;

$b$ - slip length;

$c$ - the wall thickness of double metal composite pipe.

In summary, the decline in the displacement of the double metal composite pipe:

$s_{3}=-r \varepsilon+\frac{G}{16 \pi r \lambda^{4} D}\left[2-\mathrm{e}^{-\lambda x} \cos \lambda x-\mathrm{e}^{-\lambda(b-x)} \cos \lambda(b-x)\right]$

\section{CONTACT ANALYSIS}

\section{FINITE ELEMENT MODEL}

Fig. 4 is the contact finite element model, It contains a top view and a front cutaway view. In this figure, $a$ is slip seat, $b$ is double metal composite pipe, $\mathrm{c}$ is slip tooth. The slip seat is Fully-constrained, hanging load is put at the bottom of the double metal composite pipe[14,15]. 

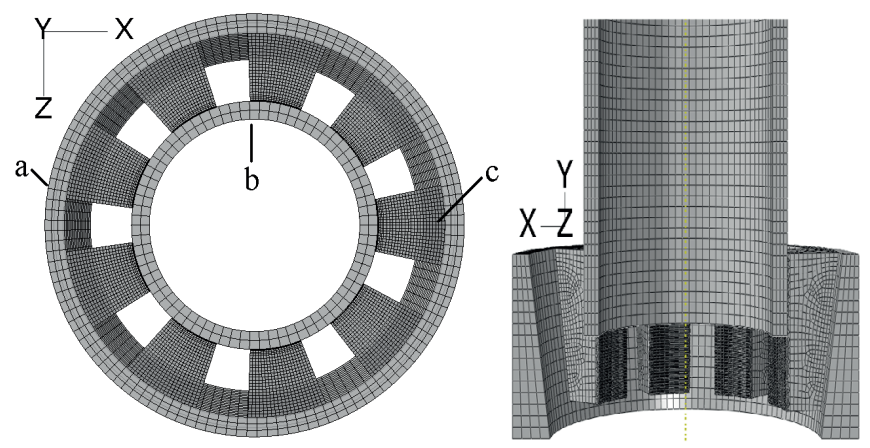

Fig. 4. Finite element model

\section{FINITE ELEMENT VERIFICATION}

From Fig. 5, There are two kinds of slip teeth plate, which was currently being used universally. the differences between them was obvious, it can be seen that there was a big difference between the contact area of the two kinds of tooth plate.

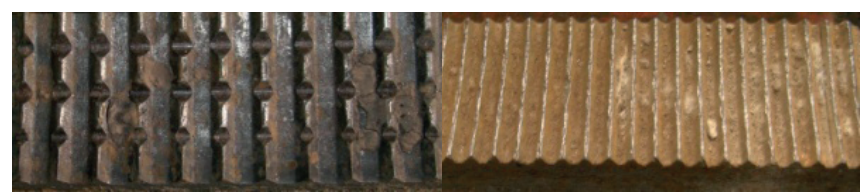

(a)

(b)

Fig. 5. Two kinds of slip teeth

With the slip clamping a double metal composite pipe of 180 tons, the elasticoplasticity contact behavior of the two slips with the double metal composite pipe was studied, the results are shown in Fig. 6. (a)

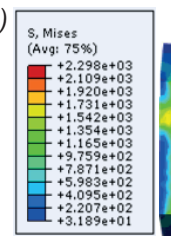

(b)

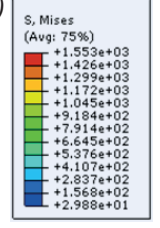

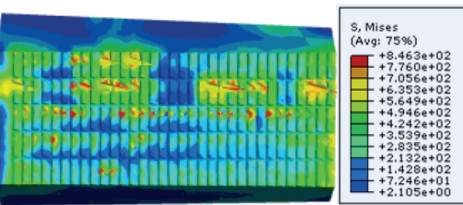
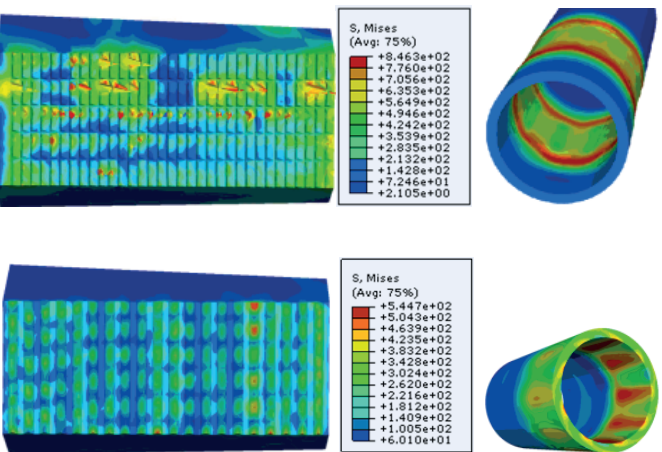

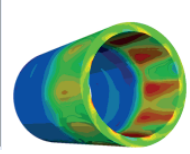

Fig. 6. The stress of teeth and double metal composite pipe

From Fig. 6, we can see that, two slip tooth plates on the double metal composite pipe damage are relatively large, there is little difference between the calculation results and field using consistent, The accuracy of the finite element model was proved by the results.

For the Eq. (8) and (19), radial load factor $B$ was determined by the slip back taper factor and friction coefficient, slip system, the existence of oil and gas fields on the current, which slips taper angle $\alpha$, monolithic slip tooth plate length $L$ and the rotation angle $\Phi$ have been fixed, and the friction coefficient $\mu$ is based on field data can be obtained, we have obtained it according Eq. (28). Simulation, we need to determine the tooth plate contact surface slip factor $A$ and the tooth top width factor $C$, thus, setting tooth rake angle changed gradually from $60^{\circ}$ to $90^{\circ}$, the tooth back angle from $30^{\circ}$ to $60^{\circ}$ gradually changed, tooth height from $0 \mathrm{~mm}$ to $0.8 \mathrm{~mm}$. After the calculations we chose the calculation results in Tab. 1 and illustrated it.

Tab. 1. Slip tooth parameters

\begin{tabular}{|c|c|c|c|c|}
\hline NO. & OA & OB & OC & OD \\
\hline$B\left(^{\circ}\right)$ & 75 & 75 & 60 & 60 \\
\hline$\gamma\left({ }^{\circ}\right)$ & 35 & 50 & 30 & 60 \\
\hline$h(\mathrm{~mm})$ & 1.8 & 0.7 & 1.3 & 2.0 \\
\hline
\end{tabular}

Now, based on the previous research, which had been published, as shown in Fig. 7, for a variety of tooth root chamfer radius change with the diameter of chamfer of tooth top, general trend of affection to stress of double metal composite pipe was the same. We planned 5 kinds of tooth root radius. There was an optimal value, where the root which has the least impact on plastic damage to the double metal composite pipe was $0.1 \mathrm{~mm}$, and the tooth top semicircle diameter is $0.25 \mathrm{~mm}[1]$.

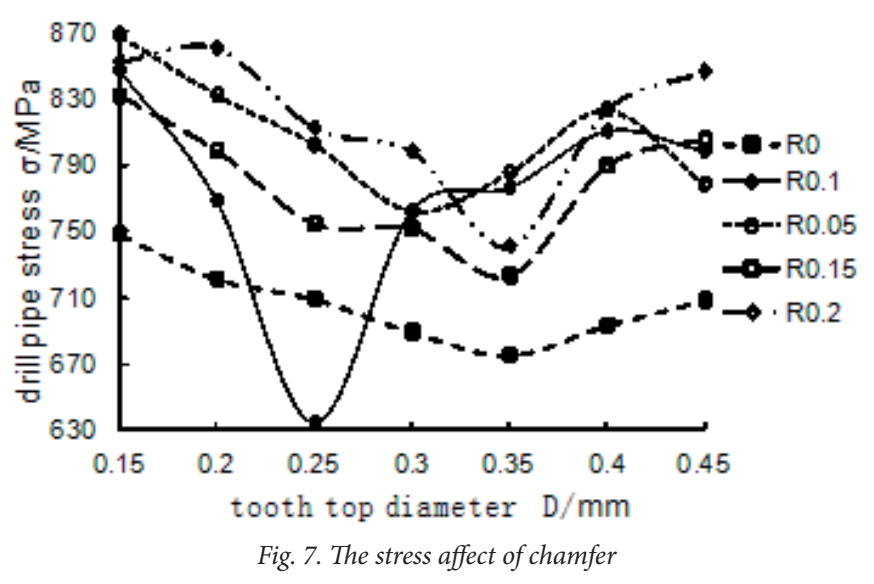

Then, author had been studied the affect of the width of tooth top and tooth root chamfer to double metal composite pipe's plastic damage, the result was shown in Fig. 8[1].

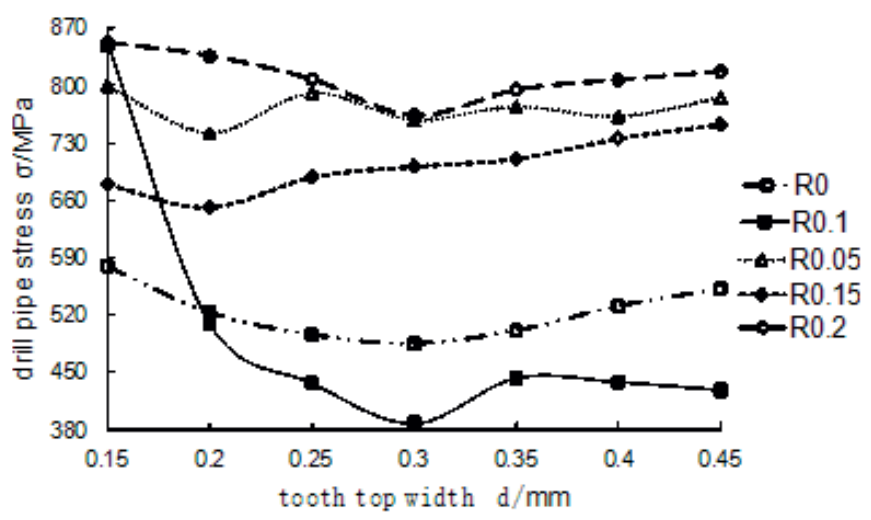

Fig. 8. The stress affect of coupling 
In Fig. 8, we knew that except when the tooth root chamfer radius was $0.1 \mathrm{~mm}$,width of tooth top was $0.15 \mathrm{~mm}$, the affect change to the double metal composite pipe was big. Under various tooth root chamfer radius, the double metal composite pipe stress change was rather flat as the tooth top width varies. Overall, when the tooth root chamfer radius was $0.1 \mathrm{~mm}$, the double metal composite pipe stress were relatively small, the best combination was that the tooth top width was $0.3 \mathrm{~mm}$, the tooth root chamfer radius was $0.1 \mathrm{~mm}[1]$.

At last, we compared the finite element results with the calculation results of Eqs., in Fig. 9 and Fig. 10, the curve was marked with finite element calculations, no mark is the result of using the derived Eqs.

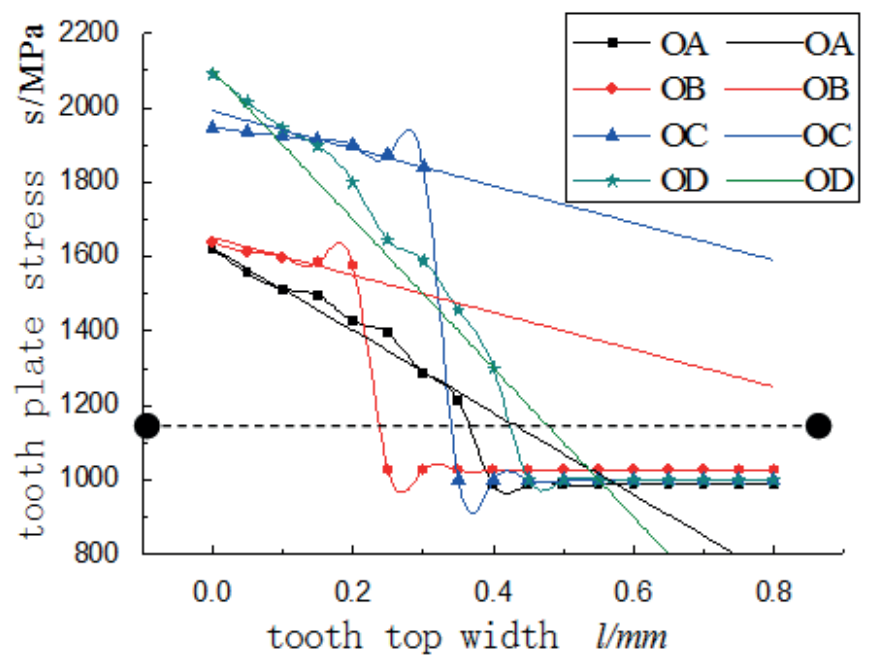

Fig. 9. Comparison of theoretical and finite element calculation results for slip tooth

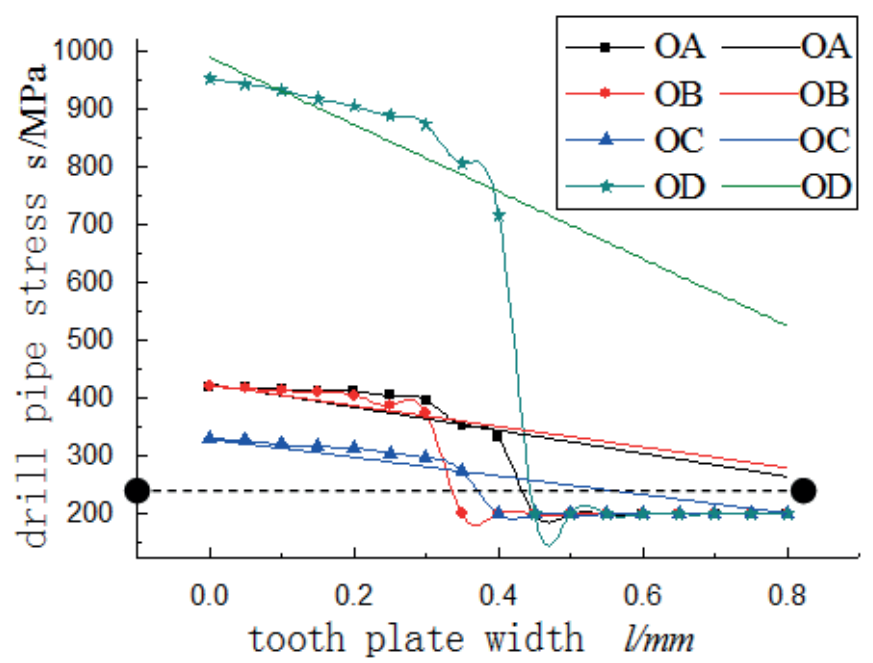

Fig. 10. Comparison of theoretical and finite element calculation results for double metal composite pipe

As can be seen from Fig. 9 and Fig. 10, the results of the finite element results and the theoretical calculation results by Eq. are basically the same above the dotted line, but below the dotted line, the calculation result was a big gap between the two, the reason is that, corresponding to different tooth width factor, in the model above the dotted line, In the finite element calculation, the double metal composite pipe has been broken off, but the theoretical Eq. was based on the calculation of the double metal composite pipe will not fall off, so there is no consistency of the results. Therefore, we can show that when slip tight pipe, the theoretical derivation is correct. We can see from Fig. 9 and Fig. 10, below the dotted line, the slip tooth stress values are the same, the reason is the value of this section, all drill has left the slip, caused exactly the same final state, so there is not gap stress basically.

All simulation results were summarized, we found that the damage and influence law of double metal composite pipe by contact area between pipe and slip tooth are the same, when the taper angle $\alpha$, chip card tile tooth plate length $\mathrm{L}$ and the rotation angle $\Phi$ were certain, the optimum contact area between pipe and tooth is the same, the optimal value is only related to the influence factor of the contact area. The contact area impact law on the double metal composite pipe as shown in Fig. 11, in the figure, strain and displacement trend was the opposite, which fits the Eq. (27).

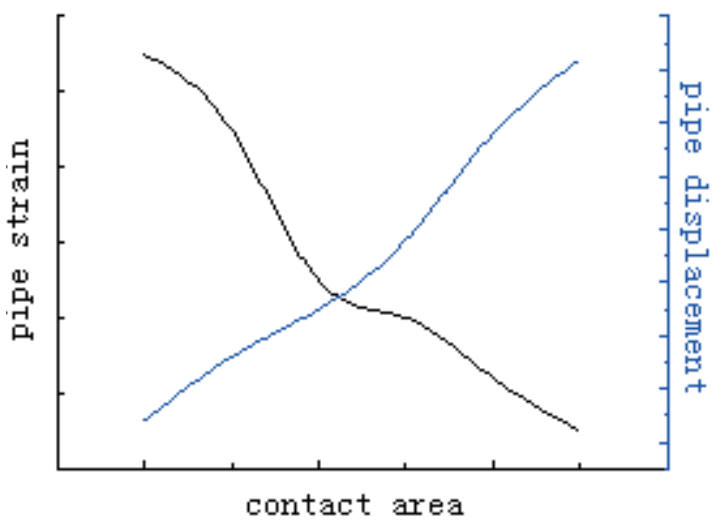

Fig. 11. Effect of contact area on double metal composite pipe

We could be obtain from Fig. 11, when we designed slip tooth, we should be get the maximum contact area based on double metal composite pipe allowable maximum displacement. Base on both theoretical and finite element, we calculated the best tooth parameters, and produced some sets of slip tooth plates, test comparison.

\section{EXPERIMENTAL VERIFICATION}

\section{EXPERIMENTAL DEVICE}

In order to better verify the theoretical analysis results, after the completion of the analysis, we produced a set of best tooth dental plate, which is $0.3 \mathrm{~mm}$, while the production of the three sets of different tooth plate test, the experimental apparatus shown in Fig. 12. 


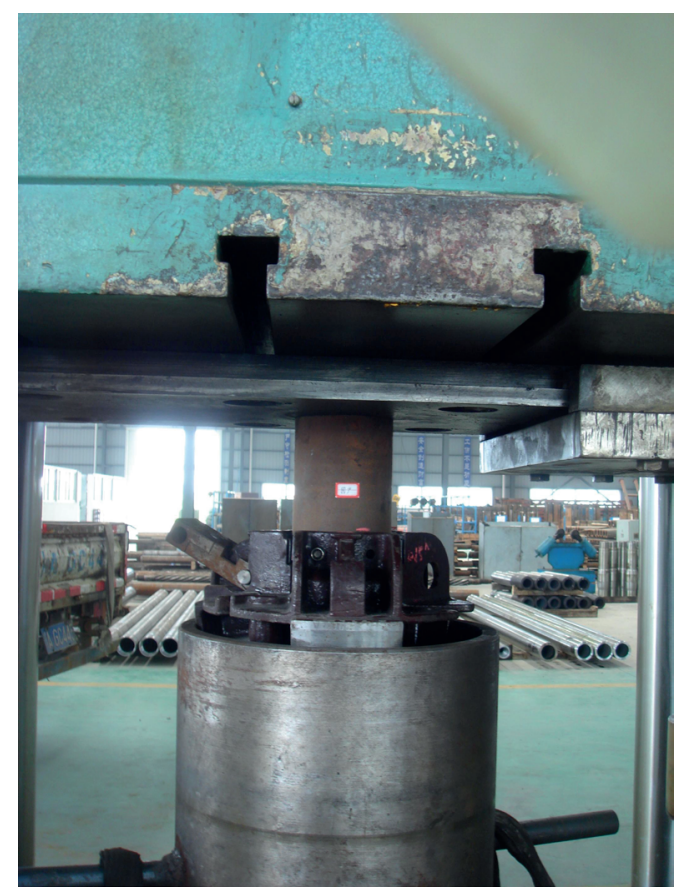

Fig. 12. Experimental device

In Fig. 12, the top is tons of hydraulic machine, the bottom of the cylinder is simulated wellhead, placed slip in the cylindrical, double metal composite pipe projected visible $150 \mathrm{~mm}$ upper the slip, invisible double metal composite pipe extends $175 \mathrm{~mm}$ in the slip, simulated wellhead apparatus is provided inside sleeve, bevel of $9^{\circ} 27^{\prime} 45^{\prime \prime} \pm 2^{\prime} 30^{\prime \prime}$, the vertical height of the sleeve is $323.8 \mathrm{~mm}$, minimum internal diameter simulated wellhead is $257.2 \mathrm{~mm}$. Observe the changes for tooth plate and double metal composite pipe after the test.

\section{EXPERIMENTAL RESULT}

First we observed the difference after test between tooth plate of designed and common tooth plate in current oil and gas fields, as shown in Fig. 13.
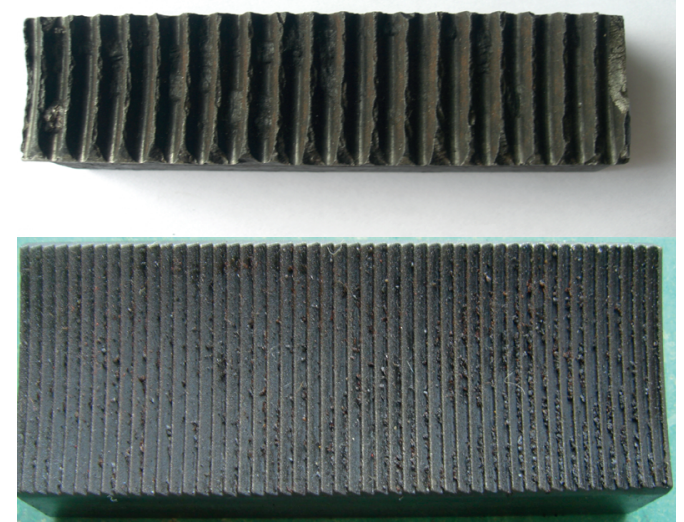

Fig. 13. Comparison of slip tooth plates
In Fig. 13, the left is currently used tooth plate in oil and gas fields, the right figure is calculated by analyzing the tooth plate, it is clear that the effect of slip tooth plate obtained by contacting the theoretical analysis area is much better than the currently used slip tooth plate.

Since we are most concerned about is the double metal composite pipe plastic damage, as shown in Fig. 14. From left to right, the first is the best slip through plastic damage tooth plate theory calculated, which is better than the other three sets of dental plate. By comparison drill Plastic Damage, we can fully proved, analysis and finite element theory for guiding the design aspects of slip tooth plate is feasible in this paper.
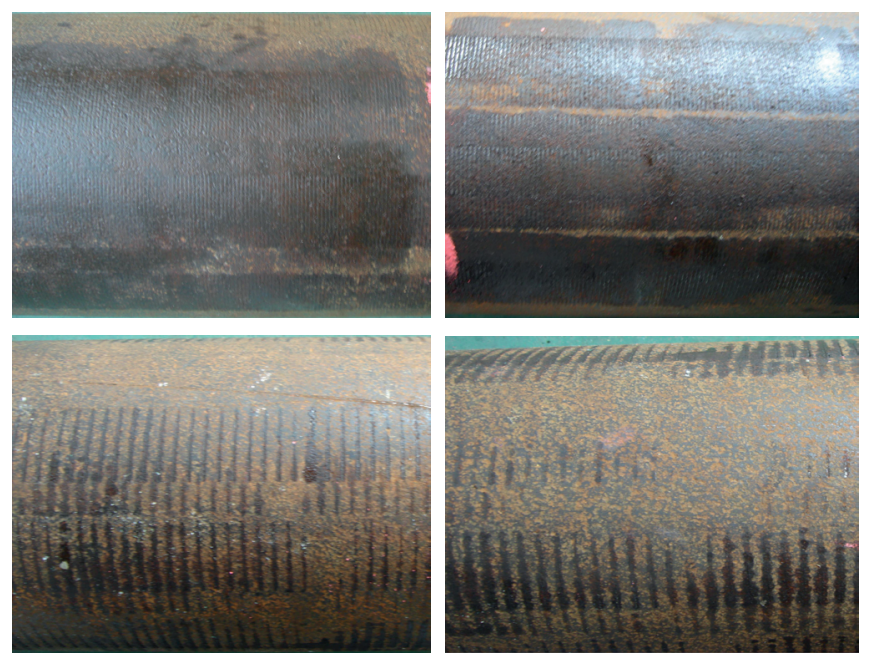

Fig. 14. Comparison of slip tooth plates

\section{CONCLUSIONS}

Impact factor of tooth top width and contact area was obtained by theoretical calculation, which provide a theoretical basis for guiding tooth designed;

The finite element model of slip and double metal composite pipe was established, then, achieved a mutual verification through simulation and finite element model;

On the basis of theory and finite element calculations, verify was completed by experimental, compared to the tooth plate and double metal composite pipe, demonstrated the results of the analysis, the best tooth top, if it is semicircle, the diameter is $0.25 \mathrm{~mm}$, if flat, width is $0.3 \mathrm{~mm}$, the study of slip system was produced a very big boost.

\section{ACKNOWLEDGEMENTS}

This work was supported by the Open Research Subject of Key Laboratory of Fluid and Power Machinery (Xihua University), Ministry of Education (Grant No. szjj2016-071). 


\section{BIBLIOGRAPHY}

1. Zhu, X.H., Wang, Y., Chen, Q.S., Deng F.C., Tang L.P.: The Elastoplastic Contact Analysis of Drilling Pipe-Slip Dog and Optimal Design of Slip Dog, The 2010 international conference on computational and information science.

2. Zhu, X.H., Wang, Y., Chen, Q.S., Tang L.P., Tong, H.: Optimal Design of Slip Dog Based on the Elasticoplasticity contact analysis, Applied Mechanics and Materials, Vol. 35, pp. 1718-1723, 2010.

3. Yeung, M.R,, Jiang, Q.H., Sun, N.: A Model of Edge2to2edge Contact for Three-dimensional Discontinuous Deformation Analysis, Computers and Geotechnics, Vol. 34, no. 3, pp. 175-186.

4. Zhou, S.P.: Calculation of the Lim ited Load-bearing Capacity of the Pipe String Inside a Slip, Journal of Xi'an Petroleum Institute(Natural Science Edition), Vol. 16, no. 2, pp. 50-54, 2001.

5. .Cui, X.B.: Analysis of the Load Bearing Capacity of Tubes Inside Slips, Acta Troleisinica, Vol. 21, no. 1, pp. 87-90, 2000.

6. Tian, H.L., Hu, S.Y.: Experimental study on frictional factor between chuck jaw and drilling rod, Coal Ceology and Exploration, Vol. 31, no. 2, pp. 68-70, 2003.

7. Liu, W.L.: To investigate the effects of different tooth surface slip on clamping force of drilling machine, Mining safety and environmental protection, Vol. 34, no. 6, pp. 81-83, 2007.

8. W.H.Spiri, W.B.Reinhold: The slip mechanism of drill pipe damage, Oil drilling machinery, no. 5, pp. 53-58, 1982.

9. Wang, Y.: The power slip nip technology of drilling tool failurel, Drilling and production technology, Vol. 29, no. 3, pp. 113-115, 2007

10. Wang, Y., Xu, W., Li, S., Zhang, H.: The Drilling Tool Against Expiration Technology of Power slip Clamp Area, Drilling \& Production Technology, Vol. 29, no. 3, pp. 113-115, 2006.

11. Merahn.,, Zayera., Shuai, B.A.: Finite element evaluation of clearance effect on tube-to-tubesheet joint strength, International Journal of Pressure Vessels and piping. Vol. 80, no, 12, pp. 579-585, 2003.

12. Liu, F.J., Zheng, J.Y.: Forming mechanism of double-layered tubes by internal hydraulic expansion, Pressure Vessel and piping, Vol. 81, no. 3, pp. 625-633, 2004.

13. Tesco Corporation: Patent Application Publication, US: 11/937,220, 2007.
14. Hibbitt., Karlsson Sorensen.: ABAQUS/ Explicit User's Manual Version6.4.1, Inc. 2003.

15. Cui, C., Luan M.T., Zhao Y.H., Sun Z.Q.: Numerical Elasto-plastic Analysis of Interaction Characteristics of PiledRaft Foundation Based on ABAQUS, Journal of Wuhan University of Technology. Vol. 31, no, 6, pp. 60-64, 2009.

\section{CONTACT WITH AUTHOR}

\author{
Wang Yu \\ e-mail:wangyu042@sohu.com \\ School of Mechatronic Engineering \\ Southwest Petroleum University \\ Chengdu Sichuan \\ 610500 \\ China
}

